\title{
Empirical Validation of Single-Room Heat Transfer Models under Uncertainty
}

\author{
Qi Li ${ }^{1}$, Ralph Muehleisen ${ }^{1}$, Baptiste Ravache ${ }^{2}$, Philip Haves ${ }^{3}$ \\ ${ }^{1}$ Energy Systems Division, Argonne National Laboratory, Lemont, IL 60439, USA \\ ${ }^{2}$ Carbon Lighthouse, San Francisco, CA 94108, USA \\ ${ }^{3}$ Building Technology and Urban Systems Division, Lawrence Berkeley National Laboratory, \\ Berkeley, CA 94720, USA
}

\begin{abstract}
This study enhances the empirical validation framework proposed by Li et al. (2017) and demonstrates it with real measurement data from a set of controlled single-room heat transfer experiments. Models were created based on the test facility and experiment descriptions, and measurement data from various types of on-site sensors. Experimental and model uncertainties were quantified and propagated based on the literature and sensor accuracy. The probabilistic absolute error (PAE) and several of its variations were developed and used as the metrics to evaluate the agreement between estimations and observations under uncertainty. Sensitivity analyses were performed to identify the influential uncertain inputs on the agreements. Results of the analysis suggest that substantial discrepancies between the current models and the measurements are present in many test scenarios and are most likely caused by remaining model assumptions, simplifications, and solution process errors that are not yet considered in the uncertainty characterization. Detailed investigations and more information and data gathering are warranted for next steps.
\end{abstract}

\section{Introduction}

Deviations of predictions of building performance simulation from the actual measured performance in real practice, widely known as the "performance gap", lead to practitioners' lack of confidence in simulation tools and lost opportunities in improving building energy efficiency and mitigating related carbon emissions. Empirical validation, which compares calculated results to monitored data from a real building, test cell, or laboratory experiment, provides an absolute truth standard for evaluating a program's ability to analyze physical behavior (ANSI/ASHRAE, 2011). However, the presence of various uncertainties in model inputs and measurements prevents previous empirical validation studies in the literature from obtaining conclusive results (Jensen 1993; Clarke, Strachan, and Pernot 1993; Lomas et al. 1997; Palomo del Barrio and Guyon 2003, 2004; Strachan et al. 2015, 2016).

The U.S. Department of Energy (DOE) funded research project, "Validation and Uncertainty Characterization for Energy Simulation: Unlocking Opportunities in Energy Efficiency", aims to address this issue by: 1) highly controlled laboratory experiments with various types of high quality, high resolution monitoring data, 2) explicit characterization and propagation of experimental and model uncertainties, and 3) use of probabilistic discrepancy metrics in comparing simulated and measured results under these uncertainties. The last two measures have been incorporated into the framework proposed by $\mathrm{Li}$ et al. (2017) and demonstrated using synthetic data. This study will enhance the framework, demonstrate it on real measurement data collected in a set of experiments for validation of single-room heat transfer models, and provide observations and discussions based on the current validation results. The actual experiments and instrumentations are described in more detail and justified in a companion paper and a recently released technical report by Haves et al. (2019a; 2019b).

\section{Methodology}

In empirical validation, the concept of an experiment not only includes the actual field experiment but also the information and data that are provided for generating a tobe-tested model. Accordingly, the adequacy of an experiment for empirical validation purpose depends both on the quality of measurement collected in the field experiment, and on whether the provided information and data can lead to a model that has reasonable agreement with the measurement. Explicit characterization and propagation of uncertainties and comparison of simulation and measurement under these uncertainties enables separation of the contributions of these uncertainties to the discrepancy between simulation and measurement from those caused by other model assumptions, simplifications, solution process errors, etc. The proposed framework, illustrated in Figure 1, not only facilitates the diagnosis and refinement of a validation experiment, but also enables detailed validation and diagnosis of a future model with an adequate experiment.

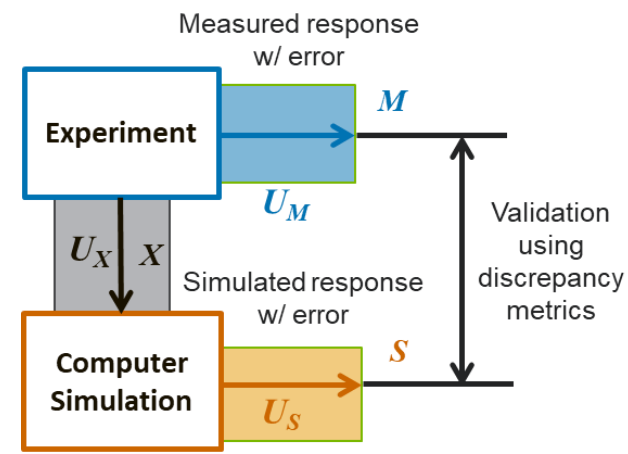

Figure 1: Illustration of the framework. 


\section{Uncertainty characterization}

The framework of Figure 1 shows the measured data, $M$, and the uncertainty in those measurements, $U_{M}$, associated with the direct measurements of physical quantities, e.g. recorded DX coil electric energy use. $U_{M}$ also includes uncertainties associated with the other quantities inferred from direct measurements, e.g. hourly system heating/cooling loads inferred from measurements of the temperature and flow rate of supply and return air.

The framework shows the building simulation model inputs, $X$, and the uncertainties in those inputs, $U_{X}$, given detailed description of the experiments and facilities. $U_{X}$ can be based on equipment specifications, standards, or research literature. For example, one specification uncertainty is the thermostat set point that comes from directly measured room air temperature. Other model uncertainties include the thermal and optical properties of most facility fabric materials (ASTM, 2012; Macdonald, 2002), and the rated performance of HVAC components (AHRI, 2001, 2009; Griffith et al., 2008).

\section{Uncertainty propagation}

Uncertainties in model inputs are propagated into the uncertainties in simulated responses $\left(U_{S}\right)$ via Monte Carlo simulation (MCS), a widely used method in uncertainty and risk analysis. Latin Hypercube design (LHD) (McKay, Beckman, and Conover, 1979) is used in the MCS to draw sample from the input space formed by these uncertainties with maximum stratification in any one-dimensional projection. In addition, to obtain a good coverage of the entire space, the minimum pairwise Euclidean distance between sample points of the sample in the input space is maximized by repeating the LHD algorithm a few times and choosing the sample that achieves this "maximin" property.

To obtain sufficiently accurate estimates of key statistics of $U_{S}$ without incurring too much computation cost, a convergence testing method with progressive LHD (Sallaberry, Helton, and Hora, 2008) is proposed to determine the appropriate sample size for the MCS. Starting from an initial LHD sample, this method sequentially doubles the sample size until the estimates converge. The progressive LHD algorithm reuses previous samples as the sample size grows while maintaining the "maximin" property.

\section{Discrepancy metrics}

For each of the measurements, two types of simulated responses are used in comparison with experiment. The first is a deterministic simulation from a single run of the model, with uncertain model parameters taking their nominal values and uncertainty in measurements ignored. The second is a probabilistic simulation where uncertainty in both simulation and measurements are propagated according to the process described above.

In addition to the commonly used normalized mean bias error (NMBE) and coefficient of variation of root mean squared error (CV-RMSE), the mean absolute error (MAE) and its normalized version (NMAE) have been developed to characterize the discrepancy between the measurements and the deterministic simulations:

$$
\begin{gathered}
M A E=\frac{1}{T} \sum_{t=1}^{T}\left|\hat{y}_{t}-y_{t}\right| \\
N M A E=\frac{M A E}{\bar{y}}, \text { with } \bar{y}=\frac{\sum_{t=1}^{T} y_{t}}{T}
\end{gathered}
$$

where $y_{t}$ and $\hat{y}_{t}$ are the measured and simulated time series responses respectively at times $t=1,2, \ldots T$. The use of absolute error (AE) ensures that errors in underand overestimation will not cancel out.

When responses like hourly system loads can be both positive (heating) and negative (cooling), $\bar{y}$ can become very small and notably inflate NMAE values. In this case, the mean of the absolute values $\overline{|y|}$ can be used in normalization, leading to the mean absolute normalized (MAN) metrics MANMAE:

$$
\text { MANMAE }=\frac{M A E}{\overline{|y|}}, \text { with } \overline{|y|}=\frac{\sum_{t=1}^{T}\left|y_{t}\right|}{T}
$$

When uncertainty is considered, a new probabilistic error measurement has been developed for comparison to ensembles of predictions. Adopted from the cumulative ranked probability score (CRPS) (Gneiting and Raftery, 2007; Li et al., 2017), the probabilistic absolute error (PAE) can be considered as a version of absolute error (AE) that compares a probabilistic simulation ensemble to a measured value. To compare time series responses, the mean probabilistic absolute error (MPAE) can be used and calculated as follows:

$$
\begin{gathered}
P A E_{t}=\int_{-\infty}^{\infty}\left(\tilde{F}_{Y_{t}}(x)-H\left(x-y_{t}\right)\right)^{2} d x \\
M P A E=\frac{1}{T} \sum_{t=1}^{T} P A E_{t}
\end{gathered}
$$

where $\tilde{F}_{Y_{t}}(x), t=1,2, \ldots T$ are the empirical cumulative distribution functions (CDFs) formed by $\widetilde{\boldsymbol{y}}_{\boldsymbol{t}}=$ $\left\{\tilde{y}_{t, 1}, \tilde{y}_{t, 2}, \ldots, \tilde{y}_{t, n}\right\}$, the probabilistic simulations of time series responses from the $n$ MCS sample points at the $t$ th time step. $H\left(x-y_{t}\right)$ is the Heaviside unit step function as defined by:

$$
H(a-b)= \begin{cases}0, & \text { if } a<b \\ 1, & \text { if } a \geq b\end{cases}
$$

As with MAE, MPAE can be similarly normalized into NMPAE and MANMPAE. In the absence of any uncertainty, the probabilistic simulation ensemble becomes a single simulation, and PAE and its variations collapse to their AE counterparts respectively.

While both measurement and simulation outputs have uncertainties, the PAE metric only considers simulation outputs to be a distribution. Therefore, the perturbedensemble method (Candille and Talagrand, 2008) to 
combine the both uncertainties into a single uncertainty for use in the PAE metric. This method is applicable as long as the measurement and simulation uncertainties can be considered uncorrelated, which is usually valid in empirical validation cases.

For actual implementation, a sample of the combined distribution of $U_{S}$ and $U_{M}$ can be obtained by the following steps:

1. Draw a sample of the original distribution of simulation outputs $U_{S}$ using standard uncertainty propagation of simulation model inputs and MCS.

2. Use each sample point in the sample of $U_{S}$ as the mean, and draw one or more sample points from $U_{M}$ around this mean. All the samples drawn in this step now form the sample of the enlarged distribution $U_{S}+U_{M}$.

The properscoring module (The Climate Corporation, 2015) is used to implement the PAE metrics in this study.

\section{Experiment diagnosis and refinement}

If the discrepancy between measurement and simulation results from a model properly created based on the information and data of the experiment remains significant according to certain criteria, the following measures can be taken to diagnose and refine the experiment's adequacy accordingly:

If measurements are mostly within the range of probabilistic simulation, but the range itself is considerably large, the dominant causes of the discrepancy are most likely within the considered uncertainties. In this case, sensitivity analysis can be used to identify uncertainties that are most influential on the discrepancy, and more information and data should be collected in the experiment to reduce those uncertainties until the discrepancy becomes satisfactory. A detailed comparison of commonly used sensitivity analysis methods can be found in Menberg et al. (2016).

If measurements mostly fall outside of the range of probabilistic simulation, the discrepancy are most likely caused by remaining model assumptions, simplifications, errors in solution process, etc. In this case, the scope of uncertainties should be expanded to include those not previously considered and could be influential on the remaining discrepancy. This process is repeated until measurements mostly fall within the range of probabilistic simulation.

\section{Model validation}

After an experiment becomes adequate through the previous procedures for at least one simulation tool, any future model created with other tools using the final set of information and data can be validated by following the same process as shown in Figure 1. This applies to both deterministic and probabilistic simulations, since the PAE metric and its variations collapse to their AE counterparts in the former case and thus make both cases comparable.

\section{Experiment and modeling}

The experiments for this study were performed at the Lawrence Berkeley National Laboratory (LBNL)
FLEXLAB test facility to validate modeling of singlezone heat transfer phenomena. The LBNL tests include 12 test scenarios performed under different weather conditions, and with varying characteristics in room thermal mass, window treatment, internal heat gain, and day/night room temperature setpoint. Because of space limitations in this paper, only 6 of the 12 scenarios with both small and large discrepancies are compared here. Full details of the entire project will be made available through a set of project reports to be released in 2019.

Table 1: Summary of FLEXLAB test scenarios.

\begin{tabular}{|c|c|c|c|c|}
\hline $\begin{array}{c}\text { Test } \\
\text { scenario }\end{array}$ & $\begin{array}{c}\text { Thermal } \\
\text { mass }\end{array}$ & $\begin{array}{c}\text { Covered } \\
\text { window }\end{array}$ & $\begin{array}{c}\text { Internal } \\
\text { load }\end{array}$ & $\begin{array}{c}\text { Room } \\
\text { setpoint }\end{array}$ \\
\hline $3: 02$ & Low & No & None & $22 / 22^{\circ} \mathrm{C}$ \\
\hline $6: 01$ & High & Yes & Varying & $26 / 26^{\circ} \mathrm{C}$ \\
\hline $11: 03$ & Low & No & None & $30 / 20^{\circ} \mathrm{C}$ \\
\hline $12: 01$ & High & No & None & $30 / 20^{\circ} \mathrm{C}$ \\
\hline $14: 01$ & High & Yes & Varying & $22 / 26^{\circ} \mathrm{C}$ \\
\hline $15: 01$ & Low & No & Varying & $30 / 20^{\circ} \mathrm{C}$ \\
\hline
\end{tabular}

Models of the test cells were created in EnergyPlus V8.9 independently from the experiment team's internal models based on the modeling documentation and experiment information the team provided, and thus representative of future to-be-tested models in other tools (i.e. would not include manually "tweaked" model inputs or hidden knowledge introduced because of facility familiarity). The boundary conditions of these models are outside weather conditions for exterior walls, the measured adjacent cell air temperature for interior/partition walls, and the measured slab under surface temperature for the floor. .

\section{Uncertainty characterization}

Table 2 summarizes the parameter uncertainties currently considered in this study, where $+/$ - means the uncertainty is represented as a range around the nominal parameter value, and \% means this range is represented as a percentage of the nominal value. For normal distributions, the $\%$ value represent \pm 3 standard deviations with additional bounding based on physical laws.

\section{Material thermal properties}

Uncertainties associated with most material thermal properties were obtained from the literature (Macdonald, 2002). As a conservative estimation, these uncertainties were assumed to follow a uniform distribution within each of the ranges. These uncertainties were applied to all opaque material in the test cells, including the equivalent layers that were created by LBNL to account for thermal bridges and construction irregularities. Because of lack of related literature, the same $\pm 5 \%$ uncertainty were also applied to the thermal resistance and conductivity of window's glazing, frame, and divider.

Optical properties of opaque material surfaces were assumed to follow a normal distribution with standard deviations from Macdonald's study. Uncertainties in optical properties of window glazing, including solar and infrared reflectance and emissivity, are assumed to follow normal distributions with a $4 \%$ standard deviation based on ASTM E903-12 (ASTM, 2012). 

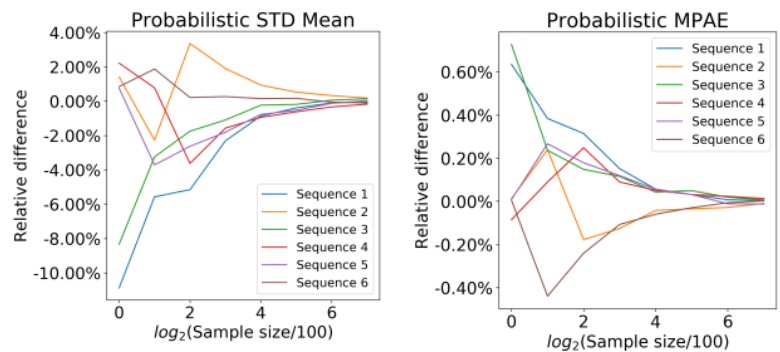

Figure 4: Convergence of the STD Mean (left) and MPAE (right) for Test Scenario 3:02

The Morris sensitivity method (Campolongo, Cariboni, and Saltelli, 2007) is used to quantify the elementary effects of experiment and model uncertainties on discrepancies. Because of the large number of uncertain inputs and the amount of repetitions recently suggested by Petersen et al. (2019) for dynamic building energy models, a two-step sensitivity analysis was performed. The first step used a Morris design with 10 repetitions and 31 levels on all 214 uncertain inputs to select a subset of 30 inputs, whose impacts were then further analyzed with a refined Morris design with 200 repetitions. This led to in total 2150 simulations in the first step and 6200 simulations in the second step for each test scenarios.

\section{Results and discussions}

Figure 5 shows the time series comparisons among the responses from deterministic simulation, the mean responses from probabilistic simulation with $95 \%$ confidence bands, and the observed responses from measurements. Table 3 summarizes the validation results of all test scenarios, using MANMAE and MANMPAE for deterministic and probabilistic simulations respectively. The mean absolute normalized versions of NMBE and CV-RMSE are also provided. It has been verified that the simulated air temperature closely matches the setpoint profiles in all test scenarios, which ensures that potential bias in comparing simulated and measured system loads.

\section{Uncertainty analysis and validation}

In general, deterministic simulations of the 6 test scenarios have an average of $-4.5 \%$ of NMBE and $45.3 \%$ of CV-RMSE, showing errors in hourly estimations that exceed the $30 \%$ criterion suggested by ASHRAE Guideline 14. The averages of MANMAE is $33.7 \%$ and close to the MANMPAE metric value of $32.8 \%$. This closeness and the relatively small uncertainty bands as observed in Figure 5 suggest that these simulation errors are likely caused by model assumptions, simplifications, and solution process errors that are unaccounted for in the current stage of uncertainty analyses.

A closer look at the result of individual scenarios shows that all discrepancy metrics vary notably among these 6 test scenarios. Scenario 3:02 appears to be the only one with satisfactory results, and significant errors are observed in Scenario 11:03, 12:01, and 15:01. While some of the estimation errors appear to be caused by irregularities in the measurements such as at the end of Scenario 14:01, the large discrepancies in other measurements are not yet clear. It was found that some

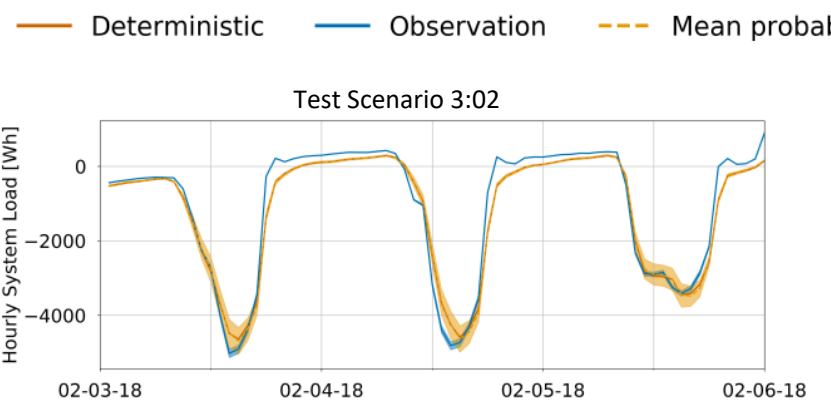

abilistic $\square$ Observation error $\square$ Full probabilistic
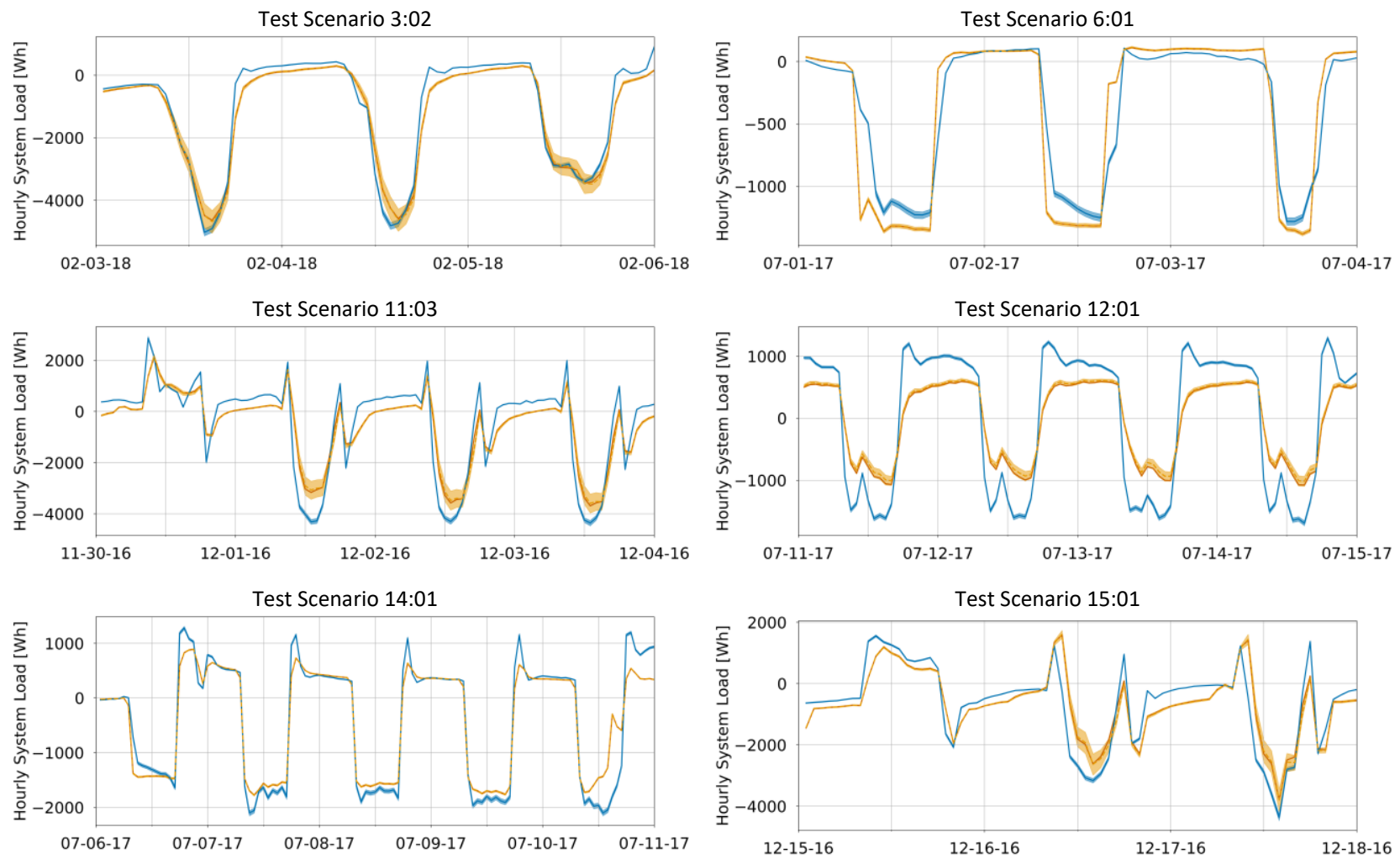

Figure 5: Comparison of hourly system loads from simulations and measurements of the 12 test scenarios. 
Table 3: Summary of FLEXLAB validation results.

\begin{tabular}{|c|c|c|c|c|}
\hline \multirow{2}{*}{$\begin{array}{c}\text { Test } \\
\text { scenario }\end{array}$} & Deterministic Simulation & Probabilistic Simulation \\
\cline { 2 - 5 } & MANMBE & MACV-RMSE & MANMAE & MANMPAE \\
\hline $3: 02$ & $-9.3 \%$ & $26.6 \%$ & $19.6 \%$ & $17.8 \%$ \\
\hline $6: 01$ & $-4.2 \%$ & $53.1 \%$ & $31.9 \%$ & $30.9 \%$ \\
\hline $11: 03$ & $-7.7 \%$ & $52.5 \%$ & $44.5 \%$ & $44.6 \%$ \\
\hline $12: 01$ & $-2.3 \%$ & $50.5 \%$ & $44.4 \%$ & $43.7 \%$ \\
\hline $14: 01$ & $0.8 \%$ & $31.0 \%$ & $19.2 \%$ & $48.5 \%$ \\
\hline $15: 01$ & $-4.2 \%$ & $58.1 \%$ & $42.8 \%$ & $32.8 \%$ \\
\hline Mean & $-4.5 \%$ & $45.3 \%$ & $33.7 \%$ & $36.2 \%$ \\
\hline Median & $-4.2 \%$ & $51.5 \%$ & $37.4 \%$ & \\
\hline
\end{tabular}

scenarios, such as 12:01, are quite sensitive to the choice of convection heat transfer algorithm, as shown in Figure 6 , and the algorithm cannot be clearly selected a priori from the current information and data. It is expected that detailed study on the measured air and surface temperature and heat flux will shed light upon this issue. In addition, recent in-depth investigation of the heat flux meter readings has found unexpected yet limited heat loss through the slab due to construction complexity. These investigations and model refinements will be the focus of follow-on research.

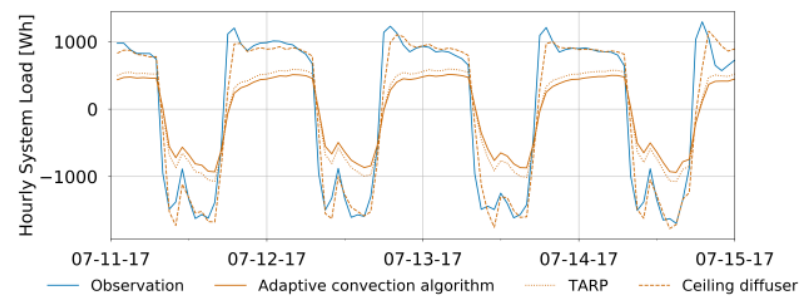

Figure 6: Comparison of deterministic simulation errors of Scenario 12:01 with different convection algorithms

In summary, the notable discrepancies between the current models and the measurements suggest that the provided information and data of the experiments are insufficient for creating models that can universally agree well with the measurements, and further collection of information and data and reexamination of uncertainties are necessary.

\section{Sensitivity analyses}

MANMAE of a deterministic simulation is used as the response in the sensitivity analyses using Morris method, where the uncertain model inputs vary one-at-a-time and repeated multiple times at different intervals of their uncertainty ranges. Metrics that reflect the influence of uncertain inputs include the mean absolute effect $\mu^{*}$, the mean effect $\mu$, and the standard deviation of effect variations $\sigma$. Detailed explanations and interpretations of these metrics can be found in Campolongo et al. (2007).

Results in Table 4 shows the top 10 inputs that are most influential on the MANMAE in each of the 6 test scenarios. Because of the different settings and boundary conditions in these scenarios, the actual ranking and contribution of each input varies. The following general observations can be obtained:

- Inputs related to room solar and infrared radiation heat gain, including measured radiation intensities, ground reflectance, glazing optical properties, etc., are consistently among the most influential inputs in these scenarios.

- The specific heat and solar/thermal absorptance of materials including plywood and gypsum board (wall constructions) and cotton batt (ceiling insulation) are also consistently influential, suggesting their impacts through thermal mass and radiant heat transfer likely with glazings.

- The magnitude of these inputs' impact on the errors are smaller than those between simulations and measurements in all scenarios.

The first two observations may mainly cause the fact that cases involving solar gains to interior surfaces tend to have more significant discrepancies between simulations and measurements. Nevertheless, the last observation suggests that factors not considered in these uncertainties are more influential and require further investigation.

\section{Conclusion}

This study enhanced the empirical validation framework proposed by Li et al. (2017) and demonstrated it with real measurement data from a set of controlled single-room heat transfer experiments. Models of the test facility were created based on the experiment information and measurement data. The framework was applied to characterize the experimental and model uncertainties and compare simulations and measurements under these uncertainties. Result of analyses suggested that considerable discrepancies between the current models and the measurements are present in most test scenarios, and are most likely caused by remaining model assumptions, simplifications, and solution process errors that are not yet considered in the uncertainty characterization.

The framework proposed and demonstrated in this study can be universally applied to future empirical validation studies to evaluate experiment adequacy. The developed probabilistic accuracy metrics can be used in various validation and calibration applications where the associated uncertainties need to be addressed. In addition, the process of uncertainty quantification and propagation demonstrated in this study can inform the inclusion of uncertainty estimates as an integral part of simulation tool output in the future. Finally, it is expected that the final information and datasets will form the basis of a supplement to the existing BESTEST tests 
Table 4: Summary of FLEXLAB sensitivity analyses results.

\begin{tabular}{|c|c|c|c|c|c|c|c|}
\hline Input & $\mu^{*}$ & $\mu$ & $\sigma$ & Input & $\mu^{*}$ & $\boldsymbol{\mu}$ & $\sigma$ \\
\hline \multicolumn{4}{|c|}{ Test Scenario 3:02 } & \multicolumn{4}{|c|}{ Test Scenario 6:01 } \\
\hline Global horizontal solar radiation & $4.2 \%$ & $-0.7 \%$ & $4.7 \%$ & Plugs schedule & $2.2 \%$ & $2.2 \%$ & $0.0 \%$ \\
\hline Glazing solar transmittance & $3.9 \%$ & $0.8 \%$ & $4.5 \%$ & Measurement error & $0.5 \%$ & $-0.3 \%$ & $0.5 \%$ \\
\hline Cotton batt 7" absorptance & $2.7 \%$ & $2.7 \%$ & $1.4 \%$ & Glazing cover infrared emissivity & $0.4 \%$ & $-0.4 \%$ & $0.1 \%$ \\
\hline Plywood absorptance & $1.6 \%$ & $-1.5 \%$ & $1.1 \%$ & Metal panel absorptance & $0.1 \%$ & $-0.1 \%$ & $0.0 \%$ \\
\hline Plywood specific heat & $1.3 \%$ & $1.3 \%$ & $0.4 \%$ & Glazing cover conductivity & $0.1 \%$ & $0.1 \%$ & $0.0 \%$ \\
\hline Gypsum board specific heat & $0.9 \%$ & $0.9 \%$ & $0.3 \%$ & Cotton batt 7" specific heat & $0.1 \%$ & $-0.1 \%$ & $0.1 \%$ \\
\hline Ground reflectance & $0.8 \%$ & $0.0 \%$ & $0.9 \%$ & Roof decking 1/2" absorptance & $0.1 \%$ & $-0.1 \%$ & $0.1 \%$ \\
\hline Polyiso specific heat & $0.6 \%$ & $0.6 \%$ & $0.4 \%$ & South wall insulation specific heat & $0.1 \%$ & $-0.1 \%$ & $0.0 \%$ \\
\hline Diffuse horizontal solar radiation & $0.6 \%$ & $0.2 \%$ & $0.6 \%$ & Topping slab absorptance & $0.1 \%$ & $-0.1 \%$ & $0.0 \%$ \\
\hline Cotton batt 7" specific heat & $0.5 \%$ & $-0.2 \%$ & $0.6 \%$ & Cement board 0.65" absorptance & $0.1 \%$ & $-0.1 \%$ & $0.0 \%$ \\
\hline \multicolumn{4}{|c|}{ Test Scenario 11:03 } & \multicolumn{4}{|c|}{ Test Scenario 12:01 } \\
\hline Glazing solar transmittance & $4.7 \%$ & $-4.7 \%$ & $0.9 \%$ & reflectance & $1.4 \%$ & $-1.4 \%$ & $0.1 \%$ \\
\hline Global horizontal solar radiation & $4.2 \%$ & $-4.2 \%$ & $0.7 \%$ & Topping slab absorptance & $1.0 \%$ & $1.0 \%$ & $0.1 \%$ \\
\hline Cotton batt 7" absorptance & $3.6 \%$ & $3.6 \%$ & $0.7 \%$ & Global horizontal solar radiat & $0.6 \%$ & $-0.6 \%$ & $0.1 \%$ \\
\hline Plywood absorptance & $2.2 \%$ & $-2.2 \%$ & $0.2 \%$ & Glazing solar transmittance & $0.6 \%$ & $0.6 \%$ & $0.1 \%$ \\
\hline Plywood specific heat & $1.7 \%$ & $1.7 \%$ & $0.2 \%$ & Glazing infrared emissivity & $0.3 \%$ & $-0.3 \%$ & $0.1 \%$ \\
\hline Cotton batt 7" specific heat & $1.2 \%$ & $1.2 \%$ & $0.2 \%$ & Cotton batt 7" absorptance & $0.3 \%$ & $-0.3 \%$ & $0.1 \%$ \\
\hline Gypsum board specific heat & $1.1 \%$ & $1.1 \%$ & $0.1 \%$ & Glazing solar reflectance & $0.2 \%$ & $0.2 \%$ & $0.0 \%$ \\
\hline Glazing infrared emissivity & $0.9 \%$ & $-0.9 \%$ & $0.4 \%$ & Roof decking 1/2" absorptance & $0.2 \%$ & $0.2 \%$ & $0.0 \%$ \\
\hline Polyiso specific heat & $0.7 \%$ & $0.7 \%$ & $0.3 \%$ & Gypsum board absorptance & $0.2 \%$ & $-0.2 \%$ & $0.0 \%$ \\
\hline Gypsum board absorptance & $0.6 \%$ & $-0.6 \%$ & $0.2 \%$ & Topping slab conductivity & $0.2 \%$ & $0.2 \%$ & $0.0 \%$ \\
\hline \multicolumn{4}{|c|}{ Test Scenario 14:01 } & \multicolumn{4}{|c|}{ Test Scenario 15:01 } \\
\hline Plugs schedule & $0.6 \%$ & $-0.6 \%$ & $0.0 \%$ & Glazing solar transmittance & $6.3 \%$ & $-6.3 \%$ & $1.2 \%$ \\
\hline Measurement error & $0.6 \%$ & $0.6 \%$ & $0.0 \%$ & Global horizontal solar radiation & $5.6 \%$ & $-5.6 \%$ & $0.9 \%$ \\
\hline Glazing cover infrared emissivity & $0.1 \%$ & $-0.1 \%$ & $0.1 \%$ & Cotton batt 7" absorptance & $3.2 \%$ & $3.2 \%$ & $0.5 \%$ \\
\hline Metal panel absorptance & $0.1 \%$ & $-0.1 \%$ & $0.0 \%$ & Plywood absorptance & $1.9 \%$ & $-1.9 \%$ & $0.4 \%$ \\
\hline Roof decking 1/2" absorptance & $0.1 \%$ & $-0.1 \%$ & $0.1 \%$ & Plywood specific heat & $1.8 \%$ & $1.8 \%$ & $0.2 \%$ \\
\hline Topping slab absorptance & $0.1 \%$ & $-0.1 \%$ & $0.0 \%$ & Gypsum board specific heat & $1.2 \%$ & $1.2 \%$ & $0.1 \%$ \\
\hline Topping slab specific heat & $0.1 \%$ & $0.1 \%$ & $0.0 \%$ & Cotton batt 7" specific heat & $1.1 \%$ & $1.1 \%$ & $0.2 \%$ \\
\hline Cement board 0.65" absorptance & $0.1 \%$ & $-0.1 \%$ & $0.0 \%$ & Glazing infrared emissivity & $1.0 \%$ & $-1.0 \%$ & $0.6 \%$ \\
\hline Topping slab conductivity & $0.1 \%$ & $-0.1 \%$ & $0.0 \%$ & Plugs schedule & $1.0 \%$ & $1.0 \%$ & $0.1 \%$ \\
\hline Gypsum board specific heat & $0.1 \%$ & $-0.1 \%$ & $0.0 \%$ & Gypsum board absorptance & $0.7 \%$ & $-0.7 \%$ & $0.2 \%$ \\
\hline
\end{tabular}

(Judkoff, Polly, Bianchi, and Neymark, 2010) that are referenced by ASHRAE Standard 140 (ASHRAE, 2002).

As stated previously, model uncertainties that are currently quantified based on the literature will be reexamined and refined if more detailed measurements become available. Methods to handle remaining influential factor, most likely in the form of model form uncertainty, will also be tested. Criteria to determine acceptable discrepancy between measurements and simulations is also suggested for future studies. Establishing the criteria to determine the validity of a model can use the same method as suggested above. Determinations based on the decision risk associated with the criteria could be another promising method, and an example can be found in Li et al. (2019).

\section{Acknowledgements}

This work was supported by the U.S. Department of Energy under Contract No. DE-AC02-06CH11357.

\section{References}

AHRI. (2001). Standard 410: Standard for Performance Rating of Forced-Circulation Air-Cooling and AirHeating Coils (1st ed.). Air Conditioning and Refrigeration Institute (AHRI). Arlington (USA).
AHRI. (2009). Standard 430: Performance Rating of Central Station Air-Handling Units. Air Conditioning and Refrigeration Institute (AHRI). Arlington (USA).

ANSI/ASHRAE. (2011). ANSI/ASHRAE 140-2011 Standard Method of Test for the Evaluation of Building Energy Analysis Computer Programs. ASHRAE. Atlanta (USA)

ASHRAE. (2002). ASHRAE Guideline 14-2002: Measurement of energy and demand savings. ASHRAE. Atlanta (USA)

ASTM. (2012). E 903 Standard test method for solar absorptance reflectance and transmittance of materials using integrating spheres (Vol. i). https://doi.org/10.1520/E0903-12.2.

Campolongo, F., Cariboni, J., and Saltelli, A. (2007). An effective screening design for sensitivity analysis of large models. Environmental Modelling \& Software 22(10), 1509-1518. https://doi.org/10.1016/j.envsoft.2006.10.004.

Candille, G., and Talagrand, O. (2008). Impact of observational error on the validation of ensemble 
prediction systems. Quarterly Journal of the Royal Meteorological Society 134(633), 959-971. https://doi.org/10.1016/j.envsoft.2006.10.004.

Clarke, J., Strachan, P. A., and Pernot, C. (1993). An approach to the calibration of building energy simulation models. Transactions of the American Society of Heating Refrigerating and Air Conditioning Engineers, 917-930.

Gneiting, T., and Raftery, A. E. (2007). Strictly proper scoring rules, prediction, and estimation. Journal of the American Statistical Association, 102(477), 359-378. https://doi.org/10.1198/016214506000001437.

Griffith, B., Long, N., Torcellini, P., Judkoff, R., Crawley, D., and Ryan, J. (2008). Methodology for modeling building energy performance across the commercial sector. National Renewable Energy Laboratory (NREL). Golden (USA)

Haves, P., Ravache, B., Fergadiotti, A., Yazdanian, M., and Kohler, C. (2019a). Accuracy of HVAC Load Predictions: Validation of EnergyPlus and DOE-2: Using an Instrumented Test Facility. Proceedings of Building Simulation 2019. Rome (Italy), 4-6 September 2019.

Haves, P., Ravache, B., Fergadiotti, A. and Yazdanian, M. (2019b), Accuracy of HVAC Load Predictions: Validation of EnergyPlus and DOE-2 using FLEXLAB. Lawrence Berkeley National Laboratory. Berkeley (USA).

Jensen, S. O. (1993). Validation of building energy simulation programs, Part I and II. Research Report PASSYS Subgroup Model Validation and Development. EUR 15115 EN. CEC. Brussels (Belgium).

Judkoff, R., Polly, B., Bianchi, M., and Neymark, J. (2010). Building Energy Simulation Test for Existing Homes (BESTEST-EX); Phase 1 Test Procedure: Building Thermal Fabric Cases. https://www1.eere.energy.gov/buildings/publicatio ns/pdfs/building america/bestest_phase1.pdf.

Li, Q., Augenbroe, G., and Muehleisen, R. (2017). A Framework for empirical validation of building performance simulation under uncertainty. Proceedings of Building Simulation 2017. San Francisco (USA). 7-9 August 2017.

Li, Q., Augenbroe, G., and Brown, J. (2019). A framework to quantify data informativeness in riskconscious building performance simulation applications. Proceedings of the 15th International Conference of the International Building Performance Simulation Association, Rome (Italy), 4-6 September 2019.

Lomas, K. J., Eppel, H., Martin, C. J., and Bloomfield, D. P. (1997). Empirical validation of building energy simulation programs. Energy and Buildings, 26(3), 253-275.
https://doi.org/10.1016/S0378--7788(97)00007-8.

Macdonald, I. (2002). Quantifying the effects of uncertainty in building simulation. University of Strathclyde. Glasgow (UK).

McKay, M. D., Beckman, R. J., and Conover, W. J. (1979). A comparison of three methods for selecting values of input variables in the analysis of output from a computer code. Technometrics, 21(2), 239. https://doi.org/10.2307/1268522.

Menberg, K., Heo, Y., and Choudhary, R. (2016). Sensitivity analysis methods for building energy models: Comparing computational costs and extractable information. Energy and Buildings, 133, 433-445.

https://doi.org/10.1016/j.enbuild.2016.10.005.

Palomo del Barrio, E., and Guyon, G. (2003). Theoretical basis for empirical model validation using parameters space analysis tools. Energy and Buildings, 35(10), 985-996. https://doi.org/10.1016/S0378-7788(03)00038-0.

Palomo del Barrio, E., and Guyon, G. (2004). Application of parameters space analysis tools for empirical model validation. Energy and Buildings, 36(1), 2333. https://doi.org/10.1016/S0378-7788(03)000392.

Petersen, S., Kristensen, M. H., and Knudsen, M. D. (2019). Prerequisites for reliable sensitivity analysis of a high fidelity building energy model. Energy and Buildings, 183, 1-16. https://doi.org/10.1016/j.enbuild.2018.10.035.

Sallaberry, C. J., Helton, J. C., and Hora, S. C. (2008). Extension of Latin hypercube samples with correlated variables. Reliability Engineering and System Safety, 93(7), 1047-1059. https://doi.org/10.1016/j.ress.2007.04.005.

Strachan P., Monari F., Kersken M., and Heusler I. (2015). IEA Annex 58: Full-scale empirical validation of detailed thermal simulation programs. Energy Procedia, 78, 3288-3293. https://doi.org/10.1016/j.egypro.2015.11.729.

Strachan, P., Svehla, K., Heusler, I., and Kersken, M. (2016). Whole model empirical validation on a fullscale building. Journal of Building Performance Simulation, 9(4), 331-350. https://doi.org/10.1080/19401493.2015.1064480.

The Climate Corporation. (2015). properscoring. https://github.com/TheClimateCorporation/propers coring. 